\title{
Cryo-Correlative Light and Electron Microscopy (Cryo-CLEM): Specimen Workflow Paths and Recent Instrument Developments
}

\author{
Michael Schwertner ${ }^{1}$, Duncan Stacey ${ }^{1}$ \\ 1. Linkam Scientific Instruments, 8 Epsom Downs, Tadworth, Surrey, KT20 5LR, United Kingdom. \\ Corresponding author: MichaelSchwertner@linkam.co.uk
}

Cryo - Correlative Light and Electron Microscopy (cryo-CLEM) is an exciting recent technique that benefits from combining the complementing advantages from Electron Microscopy (EM) and Fluorescence (Light) Microscopy (FM) [1,2,3,4]. While EM gives superior spatial resolution beyond the limit of light microscopy it mainly delivers structural information. EM has limitations in terms of the biological and chemical sensing of events. Fluorescence Microscopy (FM) on the other hand can reveal information about chemical, biological and even genetic processes and is compatible with living cells. FM markers, such as Green Fluorescent Protein (GFP), can be tailored to answer specific biological questions and are widely established. The downside of FM is that spatial resolution is severely limited compared to EM.

Cryo-CLEM brings it all together by sequentially imaging the same specimen location using both complimentary EM and FM techniques and merging the datasets. Genetic and biological events can be identified and pinpointed in FM before "zooming in" and studying details in very high resolution in EM. An elegant way to implement CLEM is to prepare biological samples in frozen-hydrated "vitrified" ice under cryo-conditions. In this state ice-crystal formation is suppressed, the biological sample can be observed in its near-native state. This has two unique advantages: First, fluorescence signals in FM are very strong under cryo-conditions, where reduced bleaching rates lead to superior signal to noise compared to imaging at room temperature. Second, frozen-hydrated samples are directly compatible with the vacuum requirements of the EM and the ultrastructural preservation of vitrified cryo-samples is considered the "gold standard". Screening in FM does not cause electron beam damage and preserves the sample for HR-EM

In this paper we will give a brief overview on the different sample preparation options, imaging workflows and recent developments in the instrumentation for cryo-CLEM.

A cryo-CLEM setup and workflow needs to fulfill the following conditions: a) The sample has to remain vitrified, e.g. kept below the critical temperature of approx. $-140{ }^{\circ} \mathrm{C} \mathrm{b}$ ) Coordinate recording and mapping is required to identify and record the sample locations of interest with both FM and EM imaging techniques c) Ice contamination which can be caused by condensation of humidity on the sample has to be prevented in all steps of sample handling and transfer. An instrument that fulfils all of the above requirements is the CMS196 cryo-correlative stage from Linkam Scientific, shown in Figure 1, inset. It uses liquid nitrogen to maintain the samples below the vitrification temperature. Up to three EM grids can be mounted and screened in one sample cassette, which is magnetically self-aligning and mounted inside the sample chamber. Several sample mount adapters and holders are available for a range of grid types and sample carriers. The sample transfer process uses a pre-cooled closed cryotransfer container and special manipulation tools. An LN2 auto-refill system allows independent operation of the system for a full day, without ice contamination. The cryo-stage is mounted on a light microscope equipped for fluorescence imaging and long working distance lenses (dry, up to NA 0.9). 
The integrated transmitted light condenser also allows standard optical contrasttechniques such as PlasDIC and / or phase contrast to be used. A high resolution motorised XY sample positioning system with optical encoders provides a calibrated sample coordinate system and enables fully automated EM grid mapping in fluorescence mode. The user can then review large area tiled / stitched fluorescence image data. The raw coordinate correlation accuracy between EM and FM in the order of one micron can be achieved, while much higher correlation accuracy below $50 \mathrm{~nm}$ can be achieved by the use of fiducial markers [5].

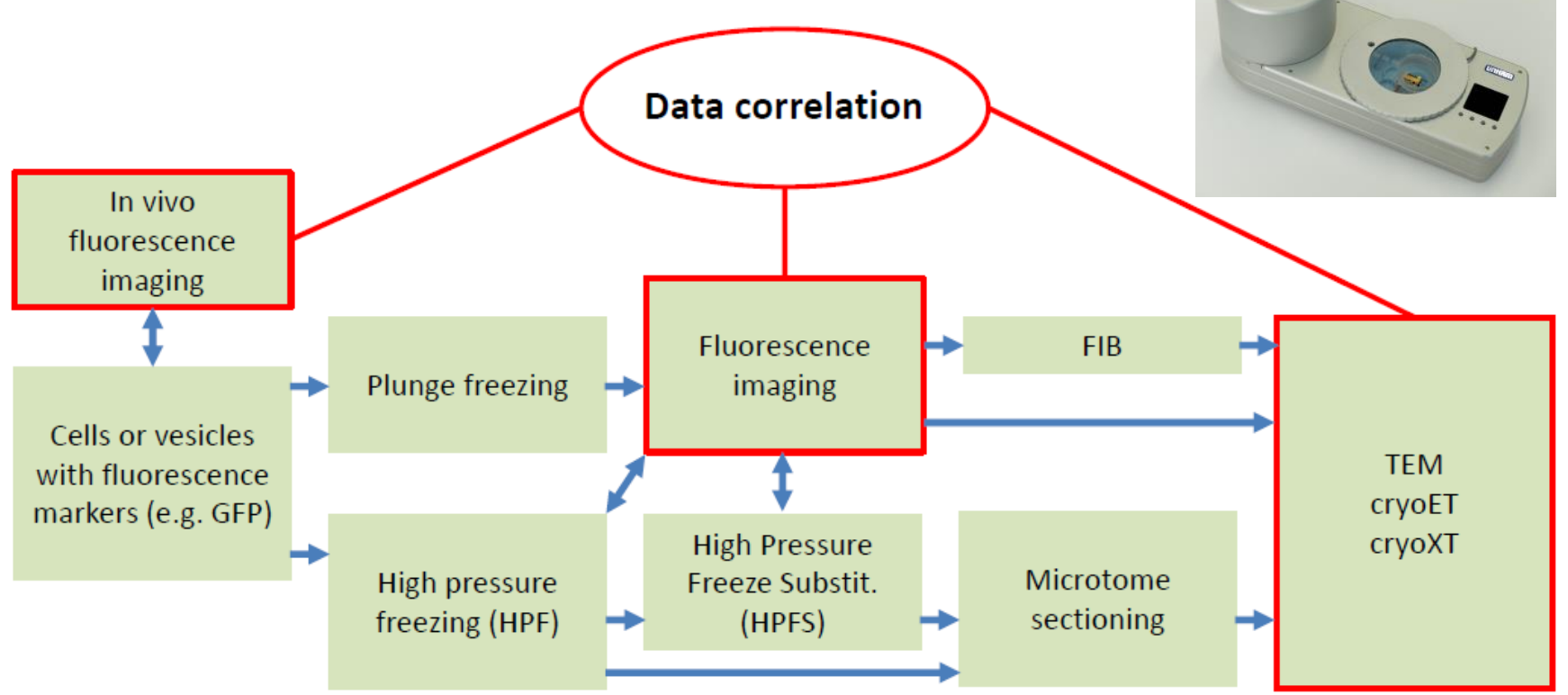

Figure 1: Flow chart with workflow options for cryo-fixation, sample processing and imaging. Inset top right: cryo-correlative stage CMS196 from Linkam Scientific for the implementation of cryo-CLEM.

The flowchart in figure 1 outlines the most common sample workflows. FM can be performed at several stages in the process. For example, cells or sub-regions can be identified during live cell imaging before cryo-fixation of the sample. Instead of EM, x-ray imaging, tomography or EM-tomography modalities can also be used for correlation with FM. It is anticipated that this young family of correlative techniques will be an indispensable tool for studying the processes of life, beyond the capabilities of FM or EM alone.

\section{References}

[1] Van Driel et al.," Tools for correlative cryo-fluorescence microscopy and cryo-electron tomography applied to whole mitochondria in human endothelial cells", EJCB No 88, Vol. 11, 621-710 [2] Briegel et al., "Correlated Light and Electron Cryo-Microscopy", Methods in Enzymology, Vol. 481, ISSN 0076-6879

[3] Methods in Cell Biology, Volume 111, Pages 1-404 (2012), Correlative Light and Electron Microscopy, Ed.Thomas Müller-Reichert \& Paul Verkade, ISBN: 978-0-12-416026-2, Academic Press [4] Methods in Cell Biology, Volume 124, Correlative Light and Electron Microscopy II, 1st Edition, (2014), Ed. Thomas Müller-Reichert and Paul Verkade, page 1 - 417, ISBN 9780128010754

[5] Schorb et al., "Correlated cryo-fluorescence and cryo-electronmicroscopy with high spatial precision and improved sensitivity", Ultramicroscopy(2013) 